\title{
INFLUÊNCIA DE DIFERENTES INGREDIENTES NA TEXTURA DE BALAS MOLES PRODUZIDAS COM E SEM GOMA GELANA
}

\author{
ANA LÚCIA FADINI * \\ FERNANDA FACCHINI ** \\ MARISE BONIFÁCIO QUEIROZ * \\ VALÉRIA DELGADO DE A. ANJOS *** \\ KATUMI YOTSUYANAGI ***
}

\begin{abstract}
Estudou-se a influência de três ingredientes, açúcar impalpável (formulação padrão), cacau em pó e polpa de morango desidratado, na firmeza de balas moles produzidas com e sem goma gelana (nome genérico do polissacarídio produzido pela fermentação controlada do microrganismo Pseudomonas elodea). Na forma nativa (high acyl), a goma gelana pode produzir géis macios e elásticos e na forma modificada (low acyl) géis duros e quebradiços. As amostras foram produzidas com a goma gelana low acyl. Cada ingrediente foi testado em três diferentes concentrações $(5$, 7 e 10\%) e adicionados na etapa de estiramento da massa de bala. As amostras foram avaliadas quanto ao parâmetro de firmeza (dureza) utilizando-se texturômetro e os resultados avaliados estatisticamente pelo teste ANOVA. As amostras também foram analisadas quanto ao teor de umidade pela metodologia de Karl Fisher. Para as balas produzidas sem goma gelana, o acréscimo na concentração dos ingredientes testados influenciou diretamente o aumento da firmeza. Já para as balas produzidas com a adição de goma gelana, o uso de $5 \%$ dos ingredientes resultou em maior firmeza e de $7 \%$ em menor firmeza. Concluiu-se que a adição de diferentes ingredientes em formulação base, com o objetivo de variar o sabor, possibilita a produção de balas com texturas distintas a partir da mesma linha de fabricação.
\end{abstract}

PALAVRAS-CHAVE: BALA MOLE; BALA-TEXTURA; GOMA GELANA.

* MSc em Tecnologia de Alimentos, Pesquisadora do Instituto de Tecnologia de Alimentos (ITAL), Centro de Pesquisa e Desenvolvimento de Chocolates, Balas, Confeitos e Panificação (CEREAL CHOCOTEC), Campinas (e-mail:fadini@ital.sp.gov.br; bqueiroz@ital.sp.gov.br).

** $\quad$ Aluna de Engenharia de Alimentos, Universidade Estadual de Campinas, e Bolsista PIBIC/CNPq (e-mail: ffacchini@hotmail.com).

*** Pesquisadores, Laboratório de Análises Físicas, Sensoriais e Estatísticas (LAFISE), ITAL, Campinas (e-mail: vanjos@ital.sp.gov.br; katumyot@ital.sp.gov.br). 


\section{INTRODUÇÃO}

Balas moles são definidas como o produto obtido pela cocção de açúcares com percentual de umidade residual entre 6-10\%. Apresentam composição semelhante à das balas duras, porém são adicionadas de gorduras e submetidas a tratamentos mecânicos (estiramento) após o cozimento até a obtenção da consistência desejada $(9,13,18)$.

De acordo com o tipo de tratamento que a massa cozida sofre e dos ingredientes que são adicionados, as balas moles podem ser classificadas em aeradas, mastigáveis e cristalizadas. As balas moles aeradas são massas de açúcares adicionadas de matérias-primas que facilitam a incorporação de ar como, por exemplo, gelatina, albumina ou ainda alguns hidrocolóides. A característica desse produto é a formação de emulsão água/óleo com suspensão de bolhas de ar. As balas moles mastigáveis podem ser adicionadas de gelatina, goma acácia, entre outros ingredientes que conferem à massa a característica mastigável e de dissolução lenta. As balas moles cristalizadas são adicionadas de matérias-primas que facilitam a inclusão de ar na massa e de outras que provocam a cristalização do açúcar, caracterizando-se como emulsão água/óleo (fase líquida super-saturada) e suspensão de microcristais de açúcar $(2,6,10$, 15). A adição de ingredientes como açúcar impalpável ou fondant na etapa de estiramento da massa pode causar a cristalização da fase líquida super-saturada, criando assim uma fase dispersa com microcristais de sacarose. Essa cristalização tende a reduzir a pegajosidade da bala nos dentes e a retração do produto depois da moldagem, alterando significativamente sua textura. Na maioria dos processos industriais isso é feito com a adição de retalhos que já sofreram cristalização, porém a adição de açúcar impalpável ou fondant possibilita cristalização mais controlada $(2,9,10,12,15)$.

Ingredientes como castanhas, cacau em pó e coco ralado, entre outros podem ser adicionados à massa de balas com o objetivo de melhorar seu sabor. No entanto, assim como o açúcar impalpável, podem causar cristalização da massa (mudam o equilíbrio da fase líquida), sendo que cada um pode influenciar de maneira diferente a textura do produto final (2).

Goma gelana é o nome genérico do polissacarídio produzido pela fermentação controlada do microrganismo Pseudomonas elodea. Na forma nativa ( $h i g h$ acyl), a goma gelana pode produzir géis macios e elásticos e na forma modificada (low acyl) géis duros e quebradiços. No que diz respeito 
às propriedades sensoriais, a água liberada durante a mastigação provoca sensação de fusão na boca. Isso contribui para que a liberação de sabor se sobressaia como uma das principais propriedades do gel $(4,11,17)$.

A goma gelana constitui agente geleificante relativamente novo, com aprovação de uso recente no Brasil. Como a aplicação dessa goma em produtos alimentícios ainda é restrita, suas propriedades não são completamente conhecidas, devendo ser estudadas sempre que houver a necessidade ou a possibilidade de sua utilização em novos produtos (3). Na linha de confeitos, em geral, a gelana pode propiciar a fabricação de produtos mais macios devido à substituição total ou parcial de outras gomas (sem nenhuma alteração no processamento), mas com o benefício da estabilidade ácida no processo $(3,16)$.

Neste trabalho avaliou-se a influência de diferentes ingredientes, adicionados na fase de estiramento, na característica de firmeza de balas moles produzidas com e sem goma gelana.

\section{MATERIAL E MÉTODOS}

As balas foram formuladas com sacarose, xarope de milho 40DE, gordura vegetal hidrogenada, emulsificante (lecitina de soja) e sal. A goma gelana testada foi a low acyl.

Para melhor padronização dos resultados da análise de textura foram produzidos dois lotes de bala, sendo um adicionado de $0,5 \%$ de goma gelana. Cada lote foi então dividido em três porções, sendo adicionados diferentes ingredientes a cada uma na fase de estiramento da massa. Foram usados açúcar impalpável (formulação padrão), cacau em pó e polpa de morango desidratada nas concentrações de 5, 7 e 10\%.

O processo de fabricação das balas seguiu o fluxograma apresentado na Figura $1(13,14)$.

As amostras foram avaliadas quanto ao parâmetro de firmeza (dureza) utilizando-se texturômetro TA-XT2/SMS nas seguintes condições:

Velocidade de pré-teste $-0,5 \mathrm{~mm} / \mathrm{s}$.

Velocidade de teste $-0,5 \mathrm{~mm} / \mathrm{s}$.

Velocidade de pós-teste - $10,0 \mathrm{~mm} / \mathrm{s}$.

Probe cilíndrico de alumínio $-\varnothing=1 / 4$ ".

Distância de compressão - 2 mm. 
Os resultados foram avaliados estatisticamente pelo teste ANOVA (5).

As amostras também foram analisadas quanto ao teor de umidade pelo método de Karl Fisher (1).

\section{FIGURA 1 - FLUXOGRAMA GERAL DE PROCESSAMENTO DAS AMOSTRAS DE BALAS MOLES}

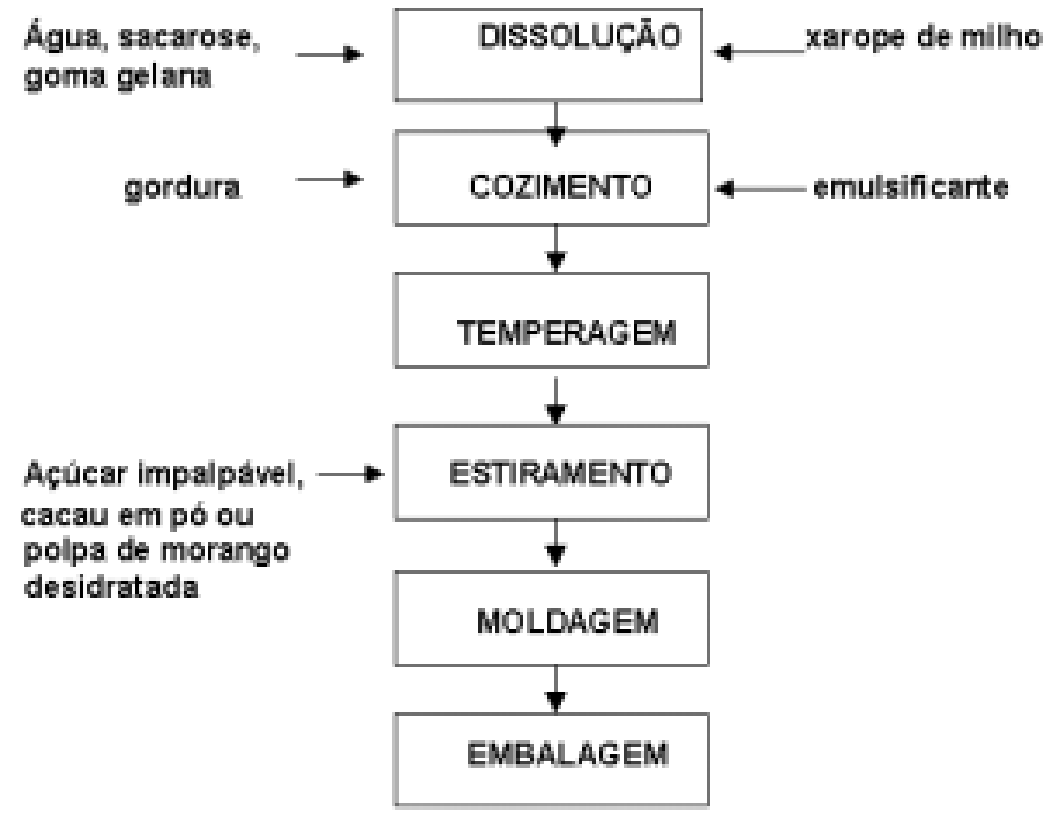

\section{RESULTADOS E DISCUSSÃO}

Na Tabela 1 estão apresentados os teores de umidade dos dois lotes de balas produzidos.

$\mathrm{Na}$ Tabela 2 constam os resultados de firmeza para as balas produzidas com e sem goma gelana, adicionadas de diferentes ingredientes em três concentrações. 
TABELA 1 - TEOR DE UMIDADE DOS DOIS LOTES DE BALAS PRODUZIDOS

\begin{tabular}{l|l|l}
\hline & $0,5 \%$ goma gelana & Sem goma gelana \\
\hline UMIDADE $(\%)$ & 8,9 & 7,9 \\
\hline
\end{tabular}

\section{TABELA 2 - COMPARAÇÃO DA FIRMEZA (g) ENTRE AS AMOSTRAS PRODUZIDAS COM E SEM GOMA GELANA}

\begin{tabular}{|c|c|c|c|}
\hline Ingredientes & $\begin{array}{c}\text { Adi } \stackrel{a}{0} 0 \\
\%\end{array}$ & $\begin{array}{c}\text { Balas produzidas com goma } \\
\text { gelana }\end{array}$ & $\begin{array}{c}\text { Balas produzidas sem } \\
\text { goma gelana }\end{array}$ \\
\hline \multirow{3}{*}{ 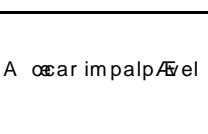 } & 5 & $6.371,13^{\mathrm{a}}$ & $1.603,72^{\mathrm{b}}$ \\
\hline & 7 & $1.199,10^{b}$ & $4.670,20^{a}$ \\
\hline & 10 & $3.258,00^{\mathrm{b}}$ & $11.676,00^{\mathrm{a}}$ \\
\hline \multirow{3}{*}{ Cacau em $p$} & 5 & $16.171,60^{\mathrm{a}}$ & $3.208,50^{\mathrm{D}}$ \\
\hline & 7 & $7.785,00^{\mathrm{a}}$ & $3.024,50^{b}$ \\
\hline & 10 & $11.754,00^{b}$ & $17.408,80^{\mathrm{a}}$ \\
\hline \multirow{3}{*}{$\begin{array}{l}\text { Polpa de morango } \\
\text { desidratada }\end{array}$} & 5 & $13.058,40^{\mathrm{a}}$ & $5.116,70^{5}$ \\
\hline & 7 & $2.594,30^{b}$ & $5.269,30^{a}$ \\
\hline & 10 & $5.232,00^{\mathrm{b}}$ & $19.603,00^{a}$ \\
\hline
\end{tabular}

Valores com letras diferentes, na mesma linha, diferem significativamente entre si para $\mathrm{p} \leq 0,05$.

Para todas as formulações testadas houve diferença significativa ( $5 \%$ de significância) entre as amostras produzidas com e sem goma. As amostras adicionadas de goma apresentaram maior firmeza para a concentração de $5 \%$ de açúcar impalpável, 5 e $7 \%$ de cacau em pó e $5 \%$ morango desidratado.

Para as amostras produzidas sem goma gelana, de forma geral, observouse que o aumento na concentração dos três ingredientes influenciou diretamente $o$ aumento da firmeza das balas. Para as amostras produzidas com goma a adição de 7\% dos ingredientes causou menor firmeza (Figura 2).

Segundo dados da literatura, a firmeza de balas com $11 \%$ de gordura (avaliadas em texturômetro TA-XT2) foi de $200 \mathrm{~g}$ (8). No presente estudo as balas foram produzidas com aproximadamente $7 \%$ de gordura. 
De acordo com dados do software do texturômetro TA-XT2 (application study) a firmeza de confeito mastigável é de 5.620,6 g $\pm 243,3$ (7).

\section{FIGURA 2 - COMPARAÇÃO DA FIRMEZA (g) ENTRE AS AMOSTRAS PRODUZIDAS COM E SEM GOMA GELANA}

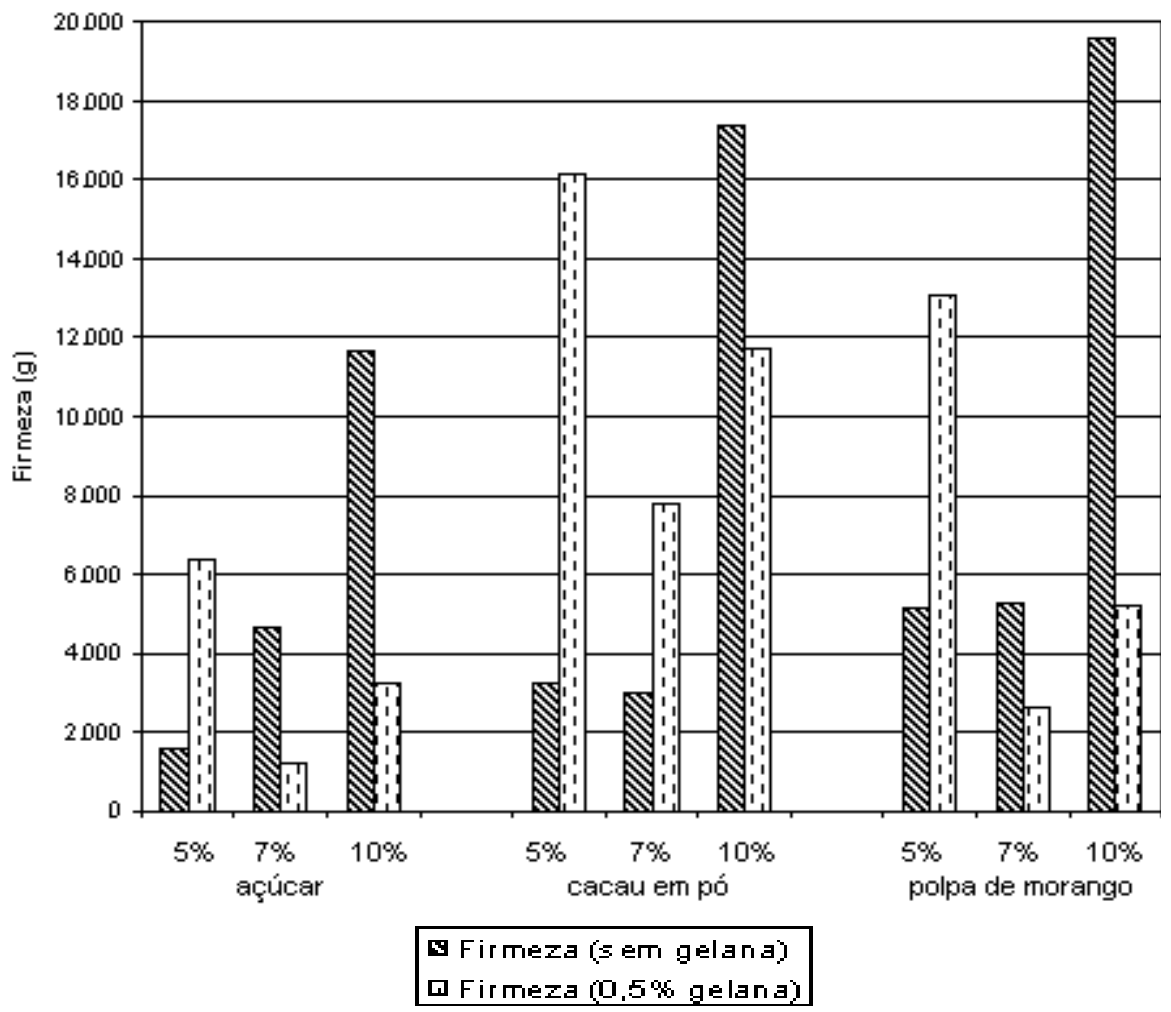

Avaliando-se a diferença entre as concentrações do mesmo ingrediente, nas balas produzidas com goma gelana (Tabela 3), verificou-se menor firmeza na adição de $7 \%$ de todos os ingredientes testados. É interessante observar que em todos os casos a concentração de $5 \%$ conferiu a maior firmeza.

Diferentemente do que ocorreu para as balas produzidas com goma gelana, a maior firmeza para as balas sem gelana foi observada com adição de $10 \%$ dos ingredientes. Nas concentrações de 5 e $7 \%$ não houve diferença significativa entre as amostras (Tabela 4). 


\section{TABELA 3 - COMPARAÇÃO DA FIRMEZA (g) ENTRE AS DIFERENTES CONCENTRAÇÕES DO MESMO INGREDIENTE PARA AS BALAS PRODUZIDAS COM GOMA GELANA}

\begin{tabular}{l|l|l|l}
\hline $\begin{array}{l}\text { Agente de } \\
\text { cristaliza a o }\end{array}$ & $5 \%$ & $7 \%$ & $10 \%$ \\
\hline A car impalp/伥 & $6.371,10^{\mathrm{a}}$ & $1.199,10^{\mathrm{c}}$ & $3.258,00^{\mathrm{b}}$ \\
\hline Cacau em p & $16.171,60^{\mathrm{a}}$ & $7.785,00^{\mathrm{c}}$ & $11.754,00^{\mathrm{b}}$ \\
\hline $\begin{array}{l}\text { Morango } \\
\text { desidratado }\end{array}$ & $13.058,40^{\mathrm{a}}$ & $2.594,30^{\mathrm{c}}$ & $5.232,10^{\mathrm{b}}$ \\
\hline
\end{tabular}

Valores com letras diferentes, na mesma linha, diferem significativamente entre si para $\mathrm{p} \leq 0,05$.

\section{TABELA 4 - COMPARAÇÃO DA FIRMEZA (g) ENTRE AS DIFERENTES CONCENTRAÇÕES DO MESMO INGREDIENTE PARA AS BALAS PRODUZIDAS SEM GOMA GELANA}

\begin{tabular}{l|c|c|c}
\hline Ingrediente & $5 \%$ & $7 \%$ & $10 \%$ \\
\hline A œar impalp/区el & $1.604,00^{\mathrm{b}}$ & $4.670,00^{\mathrm{b}}$ & $11.676,00^{\mathrm{a}}$ \\
\hline Cacau em p & $3.208,50^{\mathrm{b}}$ & $3.024,50^{\mathrm{b}}$ & $17.408,80^{\mathrm{a}}$ \\
\hline Morango desidratado & $5.117,00^{\mathrm{b}}$ & $5.269,00^{\mathrm{b}}$ & $19.603,00^{\mathrm{a}}$ \\
\hline
\end{tabular}

Valores com letras diferentes, na mesma linha, diferem significativamente entre si para $\mathrm{p} \leq 0,05$.

Para as balas com adição de goma gelana (Tabela 5), o cacau em pó foi o ingrediente que conferiu aos produtos maior firmeza nas três concentrações testadas. Já o açúcar impalpável promoveu menor firmeza.

A polpa de morango desidratada (Tabela 6 ) foi o ingrediente que conferiu maior firmeza às balas sem goma gelana, nas três concentrações testadas. O cacau em pó a 10\% também resultou em elevada firmeza, sendo as menores firmezas obtidas com o cacau em pó a 7\% e o açúcar impalpável a $5 \%$. 


\section{TABELA 5 - COMPARAÇÃO DA FIRMEZA (g) ENTRE OS DIFERENTES INGREDIENTES PARA AS BALAS PRODUZIDAS COM GOMA GELANA}

\begin{tabular}{l|c|c|c}
\hline$\%$ de adi a o & $\begin{array}{c}\text { A car } \\
\text { impalp/t/el }\end{array}$ & $\begin{array}{c}\text { Cacau } \\
\text { em p }\end{array}$ & $\begin{array}{c}\text { Morango } \\
\text { desidratado }\end{array}$ \\
\hline 5 & $6.371,10^{\mathrm{c}}$ & $16.171,60^{\mathrm{a}}$ & $13.058,40^{\mathrm{b}}$ \\
\hline 7 & $1.199,10^{\mathrm{c}}$ & $7.785,00^{\mathrm{a}}$ & $2.594,30^{\mathrm{b}}$ \\
\hline 10 & $3.258,00^{\mathrm{c}}$ & $11.754,00^{\mathrm{a}}$ & $5.232,10^{\mathrm{b}}$ \\
\hline
\end{tabular}

Valores com letras diferentes, na mesma linha, diferem significativamente entre si para $\mathrm{p} \leq 0,05$.

\section{TABELA 6 - COMPARAÇÃO DA FIRMEZA ENTRE OS DIFERENTES AGENTES DE CRISTALIZAÇÃO PARA AS BALAS PRODUZIDAS SEM GOMA GELANA}

\begin{tabular}{l|c|c|c}
\hline$\%$ de adi a o & $\begin{array}{c}\text { A œar } \\
\text { impalp/Æ } / \text { el }\end{array}$ & Cacau em $p$ & $\begin{array}{c}\text { Morango } \\
\text { desidratado }\end{array}$ \\
\hline 5 & $1.603,70^{\mathrm{c}}$ & $3.208,50^{\mathrm{b}}$ & $5.116,70^{\mathrm{a}}$ \\
\hline 7 & $4.670,20^{\mathrm{a}}$ & $3.024,50^{\mathrm{b}}$ & $5.269,30^{\mathrm{a}}$ \\
\hline 10 & $11.676,00^{\mathrm{b}}$ & $17.409,00^{\mathrm{ab}}$ & $19.603,00^{\mathrm{a}}$ \\
\hline
\end{tabular}

Valores com letras diferentes, na mesma linha, diferem significativamente entre si para $p \leq 0,05$.

A adição de cacau em pó e de polpa de morango desidratada, no geral, aumentou a firmeza das balas em relação à formulação padrão com açúcar impalpável, nas três concentrações testadas. Apenas a formulação com $7 \%$ de cacau em pó apresentou firmeza menor que o padrão.

\section{CONCLUSÃO}

Para as balas produzidas sem goma gelana, o acréscimo na concentração dos ingredientes testados influenciou diretamente o aumento da firmeza. Já para as balas produzidas com goma gelana o uso de $5 \%$ de cada um dos ingredientes resultou em maior firmeza e o uso de $7 \%$ em menor firmeza. 
Quanto à influência de cada ingrediente adicionado, a maior firmeza para as balas com goma gelana foi conferida pelo cacau em pó e a menor pelo açúcar impalpável. Para as balas sem goma, o açúcar impalpável e o cacau em pó resultaram em produtos mais macios.

Conclui-se que a adição de diferentes ingredientes em formulação base, com o objetivo de variar o sabor, possibilita a produção de balas com texturas distintas. Isso pode viabilizar a produção de diferentes produtos a partir da mesma linha de fabricação e mesma formulação. Os resultados indicam ainda que, dependendo da concentração utilizada dos ingredientes que foram estudados, a goma gelana pode pontecializar ou minimizar a influência desses sobre a firmeza das balas moles.

\section{Abstract}

INFLUENCE OF DIFFERENT INGREDIENTS ON SOFT CANDIES TEXTURE PRODUCED WITH THE ADDITION OF GELLAN GUM

The influence of three ingredients were studied, sugar powder, cocoa powder and strawberry dried pulp in the hardness of soft candies produced with and without the addition of gellan gum (generic name for the polysaccharide obtained by the controlled fermentation of the microorganism Pseudomonas elodea). At its native form (high acyl) the gellan gum can produce soft and elastic gels and at its modified form (low acyl) hard and fragile gels. The samples were produced using the low acyl gellan gum. Three different concentrations from each ingredient were tested (5, 7 and 10\%) and they were added at the pulling time in the process. The hardness of the samples was measured in a texture analyzer and the results were statistically analyzed by ANOVA test. The samples had their humidity verified by the Karl Fischer methodology. For the candies produced without gellan gum, their hardness increased proportionally to the percentage of the added ingredients. For the candies produced with gellan gum, the addition of all the tested ingredients at $5 \%$ concentration resulted to the highest hardness while the $7 \%$ concentration showed the lowest hardness. It was concluded that the addition of different ingredients to the basic formulation, with the objective of varying flavor, possibilitates the production of candies with distinct textures from the same fabrication line.

KEY-WORDS: SOFT CANDY; CANDY-TEXTURE; GELLAN GUM.

\section{REFERÊNCIAS}

1 AOAC. Association of Official Analytical Chemistry. Official methods of analysis of the AOAC. $11^{\text {th }}$ ed. Washington, 1977.

2 FABRY, I. Tecnologia de producción de caramelos blandos. Alemanha, Solingen: ZDS, 1998. 51 p. (Curso Industrial de Practicas de Chocolates y Confitería).

3 GELLAN gum, multifunctional polysaccharide for gelling and texturizing. $3^{\text {rd }}$ ed. San Diego, USA: Monsanto Company, 1996. 
4 GIBSON, W. Gellan gum. In: TMESON, A.P. Thickening and gelling agents for food. London: Blackie Academic and Professional, 1992. $227 \mathrm{p}$.

5 GOMES, F.P. Estatística experimental. 6. ed. Piracicaba: Universidade de São Paulo, Escola Superior Luiz de Queiroz, 1976. p. 7-104.

6 HALL, M.N.A. The small scale manufacture of high and low boiled sweets and toffees. London: Tropical Products Institute, 1973. p. 7-15.

7 HARDNESS and stickiness measurement of chewy confectionery by probing. New York/USA: Stable Micro Systems, 1995. (Ref.: BSW2/P6).

$8 \quad$ IZZO, M.; STAHL, C.; TUAZON, M. Using cellulose gel and carrageenan to lower fat and calories in confections. Food Technology, v. 49, n.7, p. 4549, July, 1995.

9 JACKSON, E.B. Sugar confectionery manufacture. $2^{\text {nd }}$ ed. London: Chapman \& Hall, 1995. $400 \mathrm{p}$.

10 JACKSON, E.B.; LESS, R. Sugar confectionery and chocolate manufacture. $3^{\text {rd }} \mathrm{ed}$. London: Chapman \& Hall, 1992. $379 \mathrm{p}$.

11 JANSSON, P.E.; LINDENBERG, B.; SANDFORD, P.A. Structural studies of gellan gum, an extracellular polysaccharide elaborated by Pseudomonas elodea. Carbohydrate Research, v.124, p.135-139, 1983.

12 LEES, R. The manufacture of fudge. Confectionery Production, v.43, n. 12, p. 493-494, 1977.

13 LUCCAS, V. Processamento de balas duras e mastigáveis. In: ITAL. Tecnologia de fabricação de balas. São Paulo, 1999. p. 19-38. (Manual Técnico, 17).

14 MATOSEK, T. Da dosagem dos componentes até a embalagem. In: SEMINÁRIO INDUSTRIALIZAÇÃO DE BALAS DURAS E MASTIGÁVEIS, São Paulo, 1997. Anais... São Paulo: ITAL, 1997. p. 17-19.

15 MEINERS, A.; KREITEN, K.; JOIKE, H. Silesia-essenzenfabrik Gerhard Hanke K. G. Alemanha, 1984. v. 2, 887 p. (Silesia Confiserie Manual, 3).

16 NISHINARI, K. Properties of gellan gum. Osaka, Japan: Department of Food Nutrition, 1995.

17 SANDERSON, G.R.; CLARCK, R.C. Gellan gum. Food Technology, v.37, n 4, p. 63-67, 1983.

18 VISSOTTO, F.Z. Ingredientes utilizados na fabricação de balas duras e mastigáveis. In: ITAL. Tecnologia de fabricação de balas. São Paulo, 1999. p. 1-17. (Manual Técnico, 17). 